\title{
Thrombophilia testing in the era of direct oral anticoagulants
}

\author{
Authors: Jennifer Darlow ${ }^{A_{*}}$ and Holly Mould ${ }^{B_{*}}$
}

\begin{abstract}
Venous thromboembolism (VTE) is increasingly recognised in primary and secondary care practice. The arrival of direct oral anticoagulants (DOACs) has made the management of VTE easier and more convenient. Some patients established on DOACs may need screening for underlying thrombophilias as certain thrombophilic conditions are known to confer a higher thrombosis risk, although the guidelines for when and how to test for a thrombophilia, especially in a patient taking a DOAC, are unclear. This literature review aims to examine when thrombophilia screening should take place in a patient already taking a DOAC, the effect of DOACs on thrombophilia tests, and analyse whether DOACs are safe and effective in both inherited and acquired thrombophilias.
\end{abstract}

KEYWORDS: thrombophilia testing, thrombophilia, direct oral anticoagulants, DOAC, venous thromboembolism, VTE, antiphospholipid syndrome, APS, DOAC-Stop

DOI: $10.7861 /$ clinmed.2020-1008

\section{Introduction}

Thrombophilia is a condition in which the patient's blood has an increased tendency to clot, with the first presentation usually being a venous thromboembolism (VTE). ${ }^{1}$ Thrombophilias can be inherited or acquired and confer different risks of clotting depending on the type. ${ }^{1}$ Despite the increased risk of thromboses in patients with a thrombophilia, there is not a general consensus on when testing for thrombophilias should be performed. Direct oral anticoagulant (DOAC) use has seen an exponential rise over the past decade, ${ }^{1}$ yet DOACs have been shown to affect the clotting assays used to test for thrombophilias, ${ }^{1-3}$ especially those used to test for antiphospholipid syndrome (APS). ${ }^{3}$ This review evaluates the literature available on current recommendations for thrombophilia testing in relation to DOAC usage.

\section{Types of thrombophilia}

Inherited thrombophilias can be categorised into high and low risk. High-risk thrombophilias are due to inherited deficiencies in endogenous anticoagulants, including deficiencies of

Authors: ${ }^{A}$ haematology registrar, Manchester Royal Infirmary, Manchester, UK; ${ }^{B}$ medical student, University of Manchester, Manchester, UK; *equal first authors
Protein C (PC), Protein S (PS) and antithrombin (AT), and also combined thrombophilic defects. ${ }^{4}$ Other examples include paroxysmal nocturnal haemoglobinuria (PNH) and patients with JAK2-positive myeloneoplasms. ${ }^{5}$ Low-risk thrombophilias include Factor $\mathrm{V}$ Leiden (FVL) mutation and prothrombin time (PT) G20210A gene mutations. ${ }^{4}$ Elevated factor VIII, IX and XI levels; plasminogen activator inhibitor; dysfibrinogenaemia and hyperhomocysteinaemia are also examples of low-risk thrombophilias, but are not routinely tested for in a thrombophilia screen $^{2,3,6}$ and are therefore not discussed in detail here. APS is an acquired thrombophilia and confers a high risk for both VTE as well as arterial thrombosis. ${ }^{6}$ It is characterised by the presence of lupus anticoagulant (LA), beta-2 glycoprotein and anticardiolipin antibodies. APS patients who have all three antibodies have the highest VTE risk. ${ }^{4}$

\section{Who needs thrombophilia testing?}

It is of general consensus that the majority of patients presenting with VTE should not be tested for a thrombophilia, ${ }^{6}$ and instead only selected patients should be tested. ${ }^{7,8}$ It has been proposed that inherited thrombophilias can be screened for through examination of family and personal history of VTE, without the need for a laboratory test. Patients with inherited thrombophilias often have key features in their history (Box 1). Connors suggested that all patients with a personal history of VTE at 50 years of age or younger, alongside a strong family history of VTE should be tested for a thrombophilia. ${ }^{6}$ However, guidelines from the National Institute for Clinical Excellence (NICE), the American Society of Haematology and American College of Chest Physicians suggest otherwise.

Box 1. Key features in the history of a patient with thrombophilia, which can be used to identify selected patients for thrombophilia testing

Key features in the history of a patient with an inherited thrombophilia

First VTE at less than 40-50 years of age

Strong family history of VTE - with family members having VTEs at less than 50 years of age Personal history of VTE in conjunction with weak provoking factors at a young age Personal history of VTE in an unusual location Personal history of recurrent VTE

Adapted from Connors $2017 .^{6}$ 


\section{Heritable thrombophilias}

The American Society of Haematology's 2013 'Choosing Wisely' campaign emphasised that patients presenting with a VTE with major transient risk factors (eg recent surgery, malignancy, pregnancy) should not be tested for a thrombophilia. ${ }^{9}$ However, the Society's most recent guidelines for management of VTE, published in 2018, do not comment on thrombophilia testing. ${ }^{10}$ Similarly, the American College of Chest Physicians does not give guidance regarding thrombophilia screening in its 2016 VTE update, ${ }^{11}$ while the British Committee for Standards in Haematology's document on testing for heritable thrombophilias published in 2012 states that 'it is not possible to give a validated recommendation as to how such patients (and families) should be selected' for testing. The same guideline fails to cover how to test for a thrombophilia when the patient is taking a DOAC. ${ }^{12}$

The International Union of Angiology's guidelines for thrombophilia testing are more detailed and specific (Box 2). However, the guidelines do not detail how to test for thrombophilia if a patient is on a DOAC.

NICE VTE guidelines suggest not offering thrombophilia testing to those receiving continuing anticoagulation therapy or who have had a provoked VTE. ${ }^{13}$ Antiphospholipid antibody testing should be considered when stopping anticoagulation in those with an unprovoked VTE, while thrombophilia testing may be offered if a patient has an unprovoked VTE with a first-degree relative who has had a VTE. Routine screening should not be offered for first-degree relatives.

NICE recommends that for unprovoked VTEs, consideration to a patient's clotting and bleeding risk should be made and shared decision making exercised with the patient. It recommends against sole use of risk scores for assessing clotting risk, but suggests using tools such as HAS-BLED score for assessing major bleeding risk ${ }^{13}$ (although HAS-BLED has not been validated in contexts outside of atrial fibrillation (AF)).

\section{Risk scores}

Regarding risk of recurrence following an unprovoked VTE, an assessment of clotting versus bleeding risk needs to be undertaken on an individual basis. Scoring systems exist to

Box 2. The International Union of Angiology's guidelines on thrombophilia testing

Patients who should be tested for a thrombophilia Patients who have their first VTE under 40 years of age Patients with oestrogen therapy or pregnancy as the sole risk factor for VTE

Patients who have their first unprovoked VTE under 60 years of age

Patients with recurrent VTE, irrespective of risk factor presence

Patients with recurrent superficial vein thrombosis in the absence of varicose veins

Patients with VTE at unusual sites under 50 years of age Patients with warfarin-induced skin necrosis and neonates with purpura fulminans not related to sepsis

Asymptomatic individuals with symptomatic first-degree relatives who have thrombophilia

Adapted from International Union of Angiology 2013. ${ }^{7}$ assess risk of clotting; however many of these - DASH, Vienna prediction model and HERDOO2 for example - do not take into account thrombophilia status, thus an accurate recurrence risk in thrombophilia patients cannot be calculated. ${ }^{6}$

Various risk scores also exist for assessing bleeding risk in patients who are anticoagulated. While NICE recommends using the HASBLED score to assess patients following a VTE, this has only been validated for $A F^{14}$ and there are conflicting assessments in the literature as to how good these scores are and how they compare to each other. ${ }^{15}$ Such scoring systems therefore may help to inform discussions with patients but should not be used in isolation to make decisions.

It is important to note when it comes to risk analysis that those patients who have had an unprovoked VTE, without an underlying risk factor, have a far higher risk of recurrence than those patients with a provoked VTE. ${ }^{16}$ NICE recommends that all patients with unprovoked VTE should have consideration of prolonged anticoagulation, following a discussion of risk benefit. NICE recommendation is that patients who have had a provoked proximal VTE or PE can consider discontinuation of anticoagulation if the provoking factor has been removed, ${ }^{13}$ provided 3 months treatment has been completed.

\section{Acquired thrombophilias}

Various guidelines also exist which consider thrombophilia testing for acquired thrombophilias. The Clinical Laboratory and Standards Institute explicitly notes in its 2014 guidance that LA testing is not recommended in patients concurrently taking a DOAC. ${ }^{17}$ The Scientific Standardisation Committee of the International Society on Thrombosis and Haemostasis published two sets of guidelines in 2009 and 2014 relating to antiphospholipid antibody testing. The 2009 guidelines suggest that testing for LA could take place following a transient transition to low-molecular-weight heparin (LMWH) when the patient is taking a vitamin $\mathrm{K}$ antagonist (VKA) like warfarin, but no specific reference to DOACs is made. ${ }^{18}$ Given that the 2014 guidelines do not make reference to DOACs or VKAs, ${ }^{8}$ one could infer that the same guidance for transitioning patients to LMWH holds true for patients taking DOACs.

\section{How do DOACs affect thrombophilia testing?}

DOACs have been shown to affect the clotting assays used to test for thrombophilias, ${ }^{1-3}$ especially those used to test for APS. ${ }^{3}$ However, these tests results appear accurate at DOAC trough levels ${ }^{19}$ - suggesting that anticoagulated patients could still be tested for a thrombophilia at the DOAC trough. ${ }^{3}$

Dilute Russell viper venom time (DRVVT) is a test often used in laboratories to assess for the presence of LA antibodies and is a key component of the activated protein $C$ resistance (APCR) assay, which can be used to test for inherited thrombophilias such as FVL deficiency. ${ }^{20}$ It is significantly affected by DOACs in a dose-dependent manner. ${ }^{2,19}$ Therefore, prolongation of DRVVT by DOACs leads to prolongation of clotting in the APCR assay.

There is variable prolongation of APCR assay in patients taking dabigatran and rivaroxaban and significant prolongation by apixaban. However, this is at a concentration level $50 \%$ higher than upper peak levels. ${ }^{2}$ Falsely low APCR results are also caused by DOACs, which leads to a false FVL-like picture. ${ }^{1}$

It has been recommended that FVL testing can be carried out by direct DNA detection methods, ${ }^{21}$ and indeed these are usually 
carried out if an APCR result is consistent with FVL deficiency. Genetic testing for FVL is not affected by patients already on DOACs, and will help prognosticate risk more accurately depending on the mutation present and whether a patient is homozygous.

Laboratory assays used to detect thrombophilias include one-stage clot-based assays or two-stage chromogenic assays. Chromogenic assay testing for PC levels and immunoassay testing for free PS levels are not affected by DOAC usage, but the same cannot be said for clot-based assays. ${ }^{2}$ Inhibitors of factor $\mathrm{Xa}$ (rivaroxaban, apixaban and edoxaban) have been shown to cause decreased antithrombin III (ATIII) levels and increased PS levels, therefore there is an increased risk of false positives and negatives on inherited thrombophilia testing for patients taking DOACs. ${ }^{1}$ To combat the effects of factor Xa inhibitors on ATIII, factor II-based chromogenic assays can be used in these patients with accurate results. The same holds true for dabigatran (a factor Ila inhibitor), where a factor X-based chromogenic assay can be used. ${ }^{2}$ However, these specialised tests are expensive to order and are not widely available. $^{19}$

Testing for JAK2 V617F mutation involves PCR methods ${ }^{22}$ which are not affected by DOAC use. PNH diagnosis is made through a series of clinical and laboratory investigations, ${ }^{23}$ which should not be affected by DOAC use.

\section{DOAC effects on APS testing}

A diagnosis of APS is made on the presence of clinical and laboratory criteria. The laboratory criteria include one of $L A$, anticardiolipin antibody of IgM or IgG, or anti- $\beta 2$-glycoprotein of IgM or IgG, each taken 12 weeks apart. ${ }^{24}$ Anticardiolipin and anti$\beta 2$-glycoprotein detection is largely unaffected by DOAC use.

Testing for the presence of LA in the DOAC era has many obstacles. Many patients who present with thrombosis are started on anticoagulation in the acute setting before thrombophilia testing is carried out. To complicate this further, if a patient is LA positive, another test needs to be carried out 12 weeks later to confirm a diagnosis, ${ }^{24}$ by which point a patient may be well established on a DOAC. British Society of Haematology (BSH) guidance states that for LA testing, two different methods of testing are required. The DRVVT should be one of these tests, and many laboratories would employ an activated partial thromboplastin time (APTT) based assay as the second test. Testing for LA using the DRVVT usually involves performing an initial screen, which, if positive, is followed by a confirmatory test to give a ratio. Both of these steps can be affected by DOAC use.

DRVVT screen values have been shown to be elevated with rivaroxaban, apixaban, edoxaban and dabigatran, as well as some effects on confirmatory testing. ${ }^{25}$ APTT testing in the laboratory relies on activated factor $\mathrm{X}$ to convert prothrombin to thrombin and start clot formation, therefore inhibition of factor $X$ by DOACs will prolong APTT. Some APTT reagents can be used which are insensitive to DOACs, but this would require collaboration with the laboratory and knowledge of which tests were available and which DOAC was used when interpreting results. $^{26}$

\section{Possible solutions to the DOAC effect on thrombophilia testing}

Given that thrombophilia tests appear to give accurate results at DOAC trough levels, ${ }^{19}$ it has been suggested that thrombophilia testing could take place at this point. ${ }^{1,3}$ However, the data on this are lacking and there would still be uncertainty in the test interpretation. Instead, anticoagulant medication could be temporarily halted. ${ }^{3}$ Only five DOAC half-lives are needed to reduce the DOAC serum concentrations to a level that they will not affect clot-based thrombophilia assays. In practice, this would mean withholding DOACs from the patient for 2 to 3 days. ${ }^{6}$ This comes with difficulties, such as increasing the risk of thrombotic event in highrisk patients.

An alternative to withholding anticoagulation treatment would be to transition the patient on to LMWH for $24-48$ hours. ${ }^{1,3,18}$ This would provide adequate anticoagulation with minimum interaction with the assays used.

However, care would need to be taken for testing a patient for suspected LA while on LMWH. Some reagents used in assays for APTT may be sensitive to LMWH at peak levels; ${ }^{27}$ so ideally, testing should occur at LMWH trough levels. Knowledge of local laboratory procedures is necessary. In practice, this can be complicated so is not often done. It is also important to note that AT levels can fall with LMWH use and therefore APTT test results may not be accurate. $^{28}$

One further solution to testing would be to test for thrombophilias before starting on a DOAC. This has some issues as often anticoagulation is started immediately following diagnosis of a thrombus and delaying treatment can be harmful, and acute thrombosis can decrease the levels of PC, PS and AT found in the plasma. $^{29}$

In the case of LA testing, it is possible to use different assays in the lab which are insensitive to DOACs. One such example is the Taipan snake venom time (TVST) which has been shown to successfully detect LA in patients on rivaroxaban. ${ }^{25}$ However, not all LA will be successfully detected and to meet criteria for diagnosis, a second test would still have to be used. Newly developed reagents have now been developed for use in APTT tests which are less sensitive to DOACs.

It should be noted, however, that APS can be diagnosed through presence of anticardiolipin antibody of IgG or IgM, or anti- $\beta 2$ glycoprotein of IgM or IgG, each taken 12 weeks apart. ${ }^{24}$ If a patient is already on a DOAC, LA testing is only likely to be useful to diagnose triple-positive APS, as this is high risk and will change management.

\section{The DOAC-Stop ${ }^{\mathrm{TM}}$}

The use of a novel agent X9904-20, or DOAC-Stop ${ }^{\text {TM }}$, which negates the effect of the DOAC on thrombophilia testing assays, has been proposed. ${ }^{1}$ The DOAC-Stop ${ }^{\mathrm{TM}}$ has been shown to adsorb rivaroxaban, apixaban, edoxaban and dabigatran from plasma dosed with these anticoagulants. The DOAC-Stop ${ }^{\mathrm{TM}}$ also demonstrates a statistically significant reduction in clotting time in plasma samples dosed with rivaroxaban and apixaban on PT, APTT and FVIII assays. ${ }^{30}$ Therefore, the DOAC-Stop ${ }^{\text {TM }}$ could potentially be used to counteract the effects of factor Xa inhibitors in anticoagulated patients in order to test for a thrombophilia.

However, use of this agent has drawbacks. While in most cases the DOAC-Stop ${ }^{\mathrm{TM}}$ allows for accurate testing on patients taking apixaban, rivaroxaban and dabigatran, inappropriate results have been observed in some patients with LA in up to $3 \% .^{31}$

At higher concentrations of rivaroxaban, apixaban ${ }^{30}$ and dabigatran, ${ }^{32}$ the effect of DOAC-Stop ${ }^{\text {TM }}$ is limited and does not fully negate the effect of the DOAC. One study also found that 
with different assays used in APCR testing, the DOAC-Stop ${ }^{\mathrm{TM}}$ had different effects on the results. ${ }^{33}$ An alternative to DOAC-Stop ${ }^{\text {TM }}$ - DOAC-Remove ${ }^{\mathrm{TM}}$ (5D-82410A) - has yielded promising early results in counteracting the effect of DOACs in patients both with and without thrombophilias. ${ }^{34}$ Either DOAC-Stop ${ }^{\text {TM }}$ or DOACRemove $^{\mathrm{TM}}$ could potentially be used to counteract the effects of factor Xa inhibitors in anticoagulated patients in order to test for a thrombophilia, but there is currently insufficient evidence as to support such use.

\section{Can we give DOACs in thrombophilia?}

The 2016 American College of Chest Physicians guidelines state that DOACs should be used in preference to VKAs to treat VTE, but do not give guidance regarding either drug class' use in thrombophilia. ${ }^{11}$ Due to the lack of evidence to support DOAC use in thrombophilia patients, clinicians tend to use VKAs to avoid unknown risks. ${ }^{4}$ Given the major advantages of DOACs over VKAs, there is an argument for assessing the evidence for DOAC safety and efficacy in certain thrombophilia patients.

There are inconsistent results regarding the use of DOAC safety and efficacy in patients with thrombophilia. A meta-analysis has looked at the safety of DOACs in various thrombophilias and compared outcomes to VKA use. It concluded that, especially for low-risk thrombophilia, DOACs were non-inferior to VKAs in terms of efficacy and safety profile. ${ }^{4}$

There is evidence of benefit of DOAC use in patients with PC, PS and AT deficiency, but the evidence is largely anecdotal. Given the availability of this anecdotal data, DOAC use in these settings is clearly increasingly prevalent, but further high-quality evidence is required to clarify this further. ${ }^{4}$

Although there is some thought that DOACs might be able to be used in a selected subgroup of patients with low-risk PNH following thrombosis, due to a lack of evidence, current clinical practice and recommendations is that a VKA should be used instead. ${ }^{23,35}$

JAK2 V617F mutations can be associated with the myeloproliferative neoplasms (MPNs) essential thrombocythaemia, polycythaemia vera and myelofibrosis. It is generally well accepted that management of thrombosis risk in these patients involves aggressive control of cardiovascular risk factors. Regarding whether DOACs can be used following a thrombosis in this context, the $\mathrm{BSH}$ recommends treatment with a VKA. ${ }^{36,37}$ While there is some evidence that DOACs may be non-inferior to VKAs for treatment of JAK2 V617-positive MPN associated thrombosis, the evidence is limited and DOAC use, while a possibility for the future, should be treated with caution. ${ }^{38}$

\section{Can we give DOACs in APS?}

In 2018, a large randomised controlled trial of warfarin versus rivaroxaban in patients with high-risk APS demonstrated increased risk of adverse events with rivaroxaban compared to warfarin, to the extent the trial had to be stopped before completion for safety reasons. ${ }^{39}$ Following this, in 2020, the BSH updated the guidelines on APS. ${ }^{40}$ For patients with triple antibody APS and VTE, treatment should be with warfarin and DOACs are contraindicated. If a patient is already established on a DOAC and does not wish to switch, a DOAC is preferable to no treatment but is not optimal care. There is a paucity of evidence of treatment of patients with double-positive APS and VTE, but treatment with a VKA is recommended as first line.

\section{Conclusion}

There are no clear conclusive guidelines regarding the timing of thrombophilia testing. Patients who have more risk factors for thrombophilia may be considered a higher priority for thrombophilia testing, yet many guidelines suggest thrombophilia testing should not take place if anticoagulation treatment is not being discontinued. Despite the evidence showing that DOACS affect the assays used to test for both inherited thrombophilias and APS, there are also no guidelines for how clinicians should test for thrombophilias in patients taking DOACs. Testing patients who are taking DOACs will alter the results of the tests used. Potential solutions to DOACs' effect on thrombophilia tests are to withhold DOAC treatment temporarily, test the patient at DOAC trough levels, transition the patient to $L M W H$, or use a DOAC-Stop ${ }^{T M}$, although the literature is scarce. VKAs remain the treatment of choice in patients with VTE-positive triple antibody-positive APS. There is some limited evidence for the safety of DOACs in secondary prevention of VTE associated with thrombophilias, but not enough to recommend treatment. Until further evidence is available, the decision for if a patient should be tested for thrombophilia, the timing of the testing and the treatment given should largely involve shared decision making with the patient, carefully weighing up risks and benefits on an individual basis.

\section{Key practice implications}

> If a patient has a provoked VTE, or anticoagulation is continuing and the patient does not have significant risk factors, thrombophilia testing is not recommended.

> Consider thrombophilia screening in patients with an unprovoked VTE who are at high risk or who you are considering stopping anticoagulation in.

> Risk scores can help inform discussions with patients about continuing anticoagulation, but should not be solely relied upon.

$>$ DOAC use affects assays used for most thrombophilia tests. Before thrombophilia testing, you must either stop the DOAC 2-3 days before testing if the patient is at low risk, or switch the patient to LMWH. Other options are not validated. LMWH may affect interpretation of some thrombophilia screen results.

> Treating patients who have a VTE and a confirmed thrombophilia with DOACs may be safe especially for low-risk thrombophilias; however, evidence is lacking.

> Vitamin K antagonists are the treatment of choice for patients with VTE-positive triple antibody antiphospholipid syndrome.

> Decisions on screening patients for thrombophilia and on anticoagulation treatment should be informed on the above, but ultimately made on an individual basis involving risk benefit discussions with the patient.

\section{Acknowledgements}

The authors would like to acknowledge Dr Jecko Thachil MD FRCPath, Department of Haematology, Manchester Royal Infirmary, Oxford Rd, Manchester for his guidance and contribution to editing.

\section{References}

1 Favaloro EJ. Danger of false negative (exclusion) or false positive (diagnosis) for 'congenital thrombophilia' in the age of anticoagulants. Clin Chem Lab Med 2019;57:873-82. 
2 Lippi G, Mattiuzzi C, Favaloro EJ. Thrombophilia testing in patients taking direct oral anticoagulants. Handle with care. Diagnosis 2014;1:311.

3 Favaloro EJ, Mohammed S, Curnow J, Pasalic L. Laboratory testing for lupus anticoagulant (LA) in patients taking direct oral anticoagulants (DOACs): potential for false positives and false negatives. Pathology 2019;51:292-300.

4 Elsebaie MAT, van Es N, Langston A, Büller HR, Gaddh M. Direct oral anticoagulants in patients with venous thromboembolism and thrombophilia: a systematic review and meta-analysis. J Thromb Haemost 2019:17:645-56.

5 Finazzi G, Rambaldi A, Guerini V, Carobbo A, Barbui T. Risk of thrombosis in patients with essential thrombocythemia and polycythemia vera according to JAK2 V617F mutation status. Haematologica 2007;92:135-6.

6 Connors JM. Thrombophilia testing and venous thrombosis. N Engl J Med 2017:377:1177-87.

7 International Union of Angiology. Prevention and treatment of venous thromboembolism (international consensus statement). Int Angiol 2013;32:111-260.

8 Devreese KMJ, Pierangeli SS, de Laat B et al. Testing for antiphospholipid antibodies with solid phase assays: guidance from the SSC of the ISTH. J Thromb Haemost 2014;12:792-5.

9 Hicks LK, Bering H, Carson KR et al. The ASH Choosing Wisely ${ }^{\circledR}$ campaign: five hematologic tests and treatments to question. Blood 2013;122:3879-83.

10 Witt DM, Nieuwlaat R, Clark NP et al. American Society of Hematology 2018 guidelines for management of venous thromboembolism: optimal management of anticoagulation therapy. Blood Adv 2018;2:3257-3291.

11 Kearon C, Akl EA, Ornelas ] et al. Antithrombotic therapy for VTE disease: CHEST guideline and expert panel report. Chest 2016;149:315-52.

12 Baglin T, Gray E, Greaves $\mathrm{M}$ et al. Clinical guidelines for testing for heritable thrombophilia. Br J Haematol 2010;149:209-20.

13 National Institute for Health and Care Excellence. Venous thromboembolic diseases: diagnosis, management and thrombophilia testing: NICE guideline [NG158]. NICE, 2020.

14 Senoo K, Lau YC, Lip GY. Updated NICE guideline: management of atrial fibrillation (2014). Expert Rev Cardiovasc Ther 2014;12:1037-40.

15 Rief P, Raggam RB, Hafner $F$ et al. Calculation of HAS-BLED score is useful for early identification of venous thromboembolism patients at high risk for major bleeding events: a prospective outpatients cohort study. Semin Thromb Hemost 2018;44:348-52.

16 Boutitie F, Pinede L, Schulman S et al. Influence of preceding length of anticoagulant treatment and initial presentation of venous thromboembolism on risk of recurrence after stopping treatment: analysis of individual participants' data from seven trials. BMJ 2011;342:d3036.

17 Clinical and Laboratory Standards Institute. Laboratory testing for lupus anticoagulant; approved guideline. CLSI, 2014.

18 Pengo V, Tripodi A, Reber G et al. Update of the guidelines for lupus anticoagulant detection. J Thromb Haemost 2009;7: 1737-40.

19 Lee LH. DOACs - advances and limitations in real world. Thromb J 2016;14:17.

20 Mohammed S, Favaloro EJ. Laboratory testing for activated protein C resistance (APCR). Methods Mol Biol 2017;1646:137-43.

21 Van Cott EM, Khor B, Zehnder JL. Factor V Leiden. Am J Hematol 2016;91:46-9.

22 Zhang Y, Zhou Y, Wang Y et al. Thrombosis among 1537 patients with JAK2V617F-mutated myeloproliferative neoplasms: risk factors and development of a predictive model. Cancer Med 2020;9:2096-105.
23 Parker $\mathrm{C}$. Update on the diagnosis and management of paroxysmal nocturnal hemoglobinuria. Hematology Am Soc Hematol Educ Program 2016;2016:208-16.

24 Keeling D, Mackie I, Moore GW, Greer IA, Greaves M, British Committee for Standards in Haematology. Guidelines on the investigation and management of antiphospholipid syndrome. $\mathrm{Br}$ ] Haematol 2012;157:47-58

25 Moore GW. Current controversies in lupus anticoagulant detection. Antibodies (Basel) 2016:5:22.

26 Gosselin R, Grant RP, Adcock DM. Comparison of the effect of the anti-Xa direct oral anticoagulants apixaban, edoxaban, and rivaroxaban on coagulation assays. Int J Lab Hematol 2016;38:505-13.

27 Ip BK, Thomson AR, Moriarty HT. A comparison of the sensitivity of APTT reagents to the effects of enoxaparin, a low-molecular weight heparin. Pathology 2001;33:347-52.

28 Durrani J, Malik F, Ali N, Jafri SIM. To be or not to be a case of heparin resistance. J Community Hosp Intern Med Perspect 2018;8:145-8.

29 Marlar RA, Gausman JN. Laboratory testing issues for protein C, protein S, and antithrombin. Int J Lab Hematol 2014;36:289-95.

30 Platton S, Hunt C. Influence of DOAC Stop on coagulation assays in samples from patients on rivaroxaban or apixaban. Int J Lab Hematol 2019;41:227-33.

31 Zabczyk M, Kopytek M, Natorska J, Undas A. The effect of DOAC Stop on lupus anticoagulant testing in plasma samples of venous thromboembolism patients receiving direct oral anticoagulants. Clin Chem Lab Med 2019;57:1374-81.

32 Kopatz WF, Brinkman HJM, Meijers JCM. Use of DOAC Stop for elimination of anticoagulants in the thrombin generation assay. Thromb Res 2018;170:97-101.

33 Favaloro EJ, Gilmore G, Bonar R et al. Laboratory testing for activated protein $C$ resistance: rivaroxaban induced interference and a comparative evaluation of andexanet alfa and DOAC Stop to neutralise interference. Clin Chem Lab Med 2020;58:1322-31.

34 Kopytek M, Ząbczyk M, Malinowski KP, Undas A, Natorska J. DOAC Remove abolishes the effect of direct oral anticoagulants on activated protein $\mathrm{C}$ resistance testing in real-life venous thromboembolism patients. Clin Chem Lab Med 2020;58:430-7.

35 Skeith L. Anticoagulating patients with high-risk acquired thrombophilias. Blood 2018;132:2219-29.

36 Harrison CN, Bareford D, Butt $\mathrm{N}$ et al. Guideline for investigation and management of adults and children presenting with a thrombocytosis. Br J Haematol 2010;149:352-75.

37 McMullin MFF, Mead AJ, Ali S et al. A guideline for the management of specific situations in polycythaemia vera and secondary erythrocytosis: a British Society for Haematology guideline. $\mathrm{Br}$ ] Haematol 2019:184:161-75.

38 Ianotto JC, Couturier MA, Galinat $\mathrm{H}$ et al. Administration of direct oral anticoagulants in patients with myeloproliferative neoplasms. Int J Hematol 2017;106:517-21.

39 Pengo V, Denas G, Zoppellaro G et al. Rivaroxaban vs warfarin in high-risk patients with antiphospholipid syndrome. Blood 2018;132:1365-71.

40 Arachchillage DRJ, Gomez K, Alikhan R et al. Addendum to British Society for Haematology guidelines on investigation and management of antiphospholipid syndrome, 2012 (Br. J. Haematol 2012; 157: 47-58): use of direct acting oral anticoagulants. $\mathrm{Br}$ ] Haematol 2020;189:212-5

Address for correspondence: Jennifer Darlow, Department of Haematology, Manchester Royal Infirmary, Oxford Road, Manchester M13 9WL, UK.

Email: jenny.wilson@doctors.org.uk 